\title{
La accesibilidad potencial y real del formato pdf: análisis de diarios digitales españoles
}

\author{
Por Ramón Voces y Lluís Codina
}

\begin{abstract}
Resumen: La accesibilidad de contenidos en internet no se limita ni a las páginas web ni al formato Xhtml. En este trabajo se argumenta que el formato pdf utilizado cada vez de forma más habitual en la web para publicar información debe ser objeto también del interés de los estudios de accesibilidad. En este sentido, se argumenta también que accesibilidad potencial no implica accesibilidad real, y se realiza un análisis de la accesibilidad real de las publicaciones en línea en formato $p d f$ de los principales diarios españoles.

Palabras clave: Accesibilidad potencial, Accesibilidad real, Pdf, Diarios españoles, Diarios en línea.

Title: Potential accessibility versus real accessibility of pdf format: Analysis of the Spanish online newspapers

Abstract: The accessibility of the internet contents are not limited nor to web pages or Xhtml format. In this work, is explained that pdf format is every time more and more used in the web to publish news information and for this reason it must be object of accessibility analysis. Is explained, too, that potential accessibility is not real accessibility, and is presented and analysis of the pdfformat used for online Spanish newspapers.

Keywords: Potential accessibility, Real accessibility, Pdfformat, Spanish newspapers, Online newspapers.

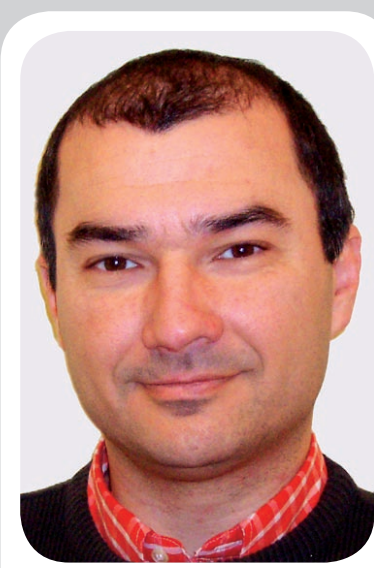

Ramón Voces es ingeniero técnico en telecomunicaciones, licenciado en documentación y diploma en estudios avanzados por la Universidad Autónoma de Barcelona. Es profesor asociado en los estudios de documentación de la $\cup A B$, miembro del Grupo de Investigación Laicom de la UAB y colaborador del grupo DigiDoc de la UPF. Sus intereses de investigación se centran en la publicación digital, el multimedia y la accesibilidad; temas de su futura tesis doctoral.

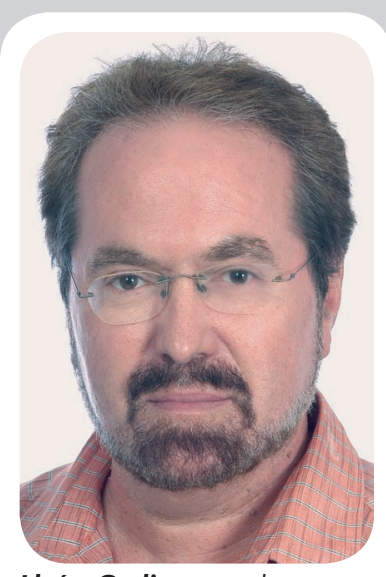

Lluís Codina es doctor en ciencias de la información, profesor de documentación en la Universidad Pompeu Fabra y coordinador del Grupo de Investigación DigiDoc del Departamento de Periodismo y de Comunicación Audiovisual de la citada universidad. Imparte docencia en los estudios de periodismo y de comunicación audiovisual de la UPF y participa en el Master interuniversitario UB/UPF sobre gestión de contenidos digitales.
\end{abstract}

Voces, Ramón; Codina, Lluís. "La accesibilidad potencial y real del formato pdf: Análisis de diarios digitales españoles". En: El profesional de la información, 2008, marzo-abril, v. 17, n. 2, pp. 205-212.

DOI: 10.3145/epi.2008.mar.11

\section{Contexto}

LA "NUEVA" SOCIEDAD DE LA INFORMACIÓN en tanto que sociedad comparte características comunes con las que nos han precedido. Por ejemplo, todas han tenido un hecho característico que ha servido de base para su desarrollo. En la sociedad industrial la invención del motor y el paso de la tracción animal a la mecánica explican muchos de los cambios que entonces se produjeron. Probablemente para la sociedad de la información el hecho diferencial sea internet, que se está convirtiendo en el "motor" y la base de los cambios actuales.
El lado oscuro de esta cuestión es una característica común a todas las sociedades: la exclusión. Siempre han existido colectivos que por una razón o por otra han quedado fuera del sistema. En la sociedad de la información la exclusión ${ }^{1}$ (en este caso digital), se manifiesta por la imposibilidad de acceder a internet. En este sentido se suelen considerar tres tipos de motivos fundamentales:

- Económicos. Tanto desde una concepción individual o de estado-nación.

- Formativos. La sociedad de la información es tecnológica y requiere conocimientos y habilidades que no todo el mundo posee.
- Fisiológicos. La barrera se produce por la imposibilidad de acceder a la información debido a limitaciones físicas o fisiológicas.

No obstante, es gratificante observar cómo en los últimos años han ido apareciendo iniciativas dirigidas a erradicar o en todo caso minimizar la exclusión digital. Por ejemplo, a nivel económico resalta la iniciativa $O L P C$ de Nicholas Negroponte, con el objetivo de proporcionar portátiles a 100 US\$, o en el campo formativo el trabajo constante de las bibliotecas en la alfabetización digital.

Además en los últimos años ha aparecido un nuevo ámbito profe- 
sional y de investigación destinado específicamente a la eliminación de la exclusión por motivos fisiológicos. Este campo se denomina habitualmente accesibilidad web. Presentarla brevemente, cuáles son sus componentes y sus tendencias actuales, es el objetivo central de este trabajo, para pasar después a ponerla a prueba en el caso de las ediciones digitales en pdf de diarios españoles.

\section{Accesibilidad web: un sistema complejo}

El organismo que publica las principales normas sobre la web (el World Wide Web Consortium) lanzó a fines de la década de los noventa la Iniciativa para la accesibilidad web (WAI o Web Accessibility Initiative). Desde entonces la WAI ha orientado la doctrina, la investigación y las principales propuestas sobre el tema, aunque sigue siendo una gran desconocida, no ya entre el gran público sino incluso entre algunos colectivos profesionales.

Ahora bien, para entenderla es útil considerarla como un sistema, como cuando nos referimos a un sistema económico, gravitatorio o digestivo; es decir, como una entidad compuesta por diferentes elementos todos ellos relacionados y cada uno con sus funciones específicas.

Por tanto, como en cualquier sistema, es importante disponer de un modelo que permita su estudio y de alguna forma prever tanto sus posibilidades como las limitaciones que conlleva.

En el caso de la accesibilidad web, normalmente se utiliza el diagrama creado por el WAI y que se reproduce en la figura 1.

Este modelo se basa en tres componentes fundamentales:

- Contenido. Establece las características que ha de tener un contenido para ser accesible.

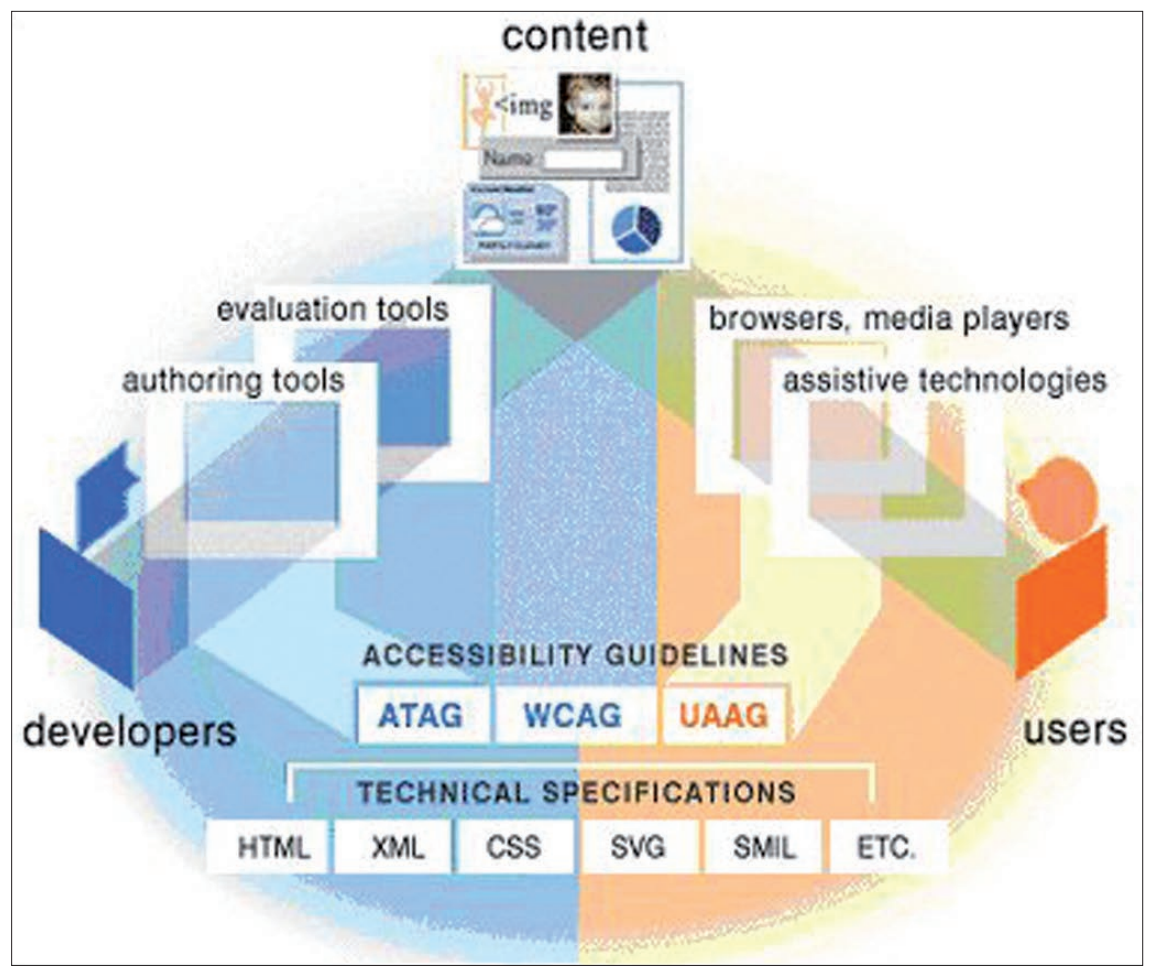

Figura 1. Componentes de la accesibilidad web Fuente: http://www.w3.org/WAl/intro/components-desc.html\#guide

- Desarrollo. Constituye la forma mediante la cual un desarrollador debería producir el contenido accesible.

- Usuario. Indica la forma mediante la cual un usuario tendría que consumir un contenido accesible.

\section{Accesibilidad potencial vs. accesibilidad real}

Conseguir que un contenido web sea accesible ni es un proceso trivial ni es automático (al menos en la mayoría de ocasiones). Desde el punto de vista técnico es necesario que se cumplan 3 requisitos:

- La tecnología utilizada debe ser accesible. Esto es, debe estar diseñada para contener o dar cabida a todos los elementos de accesibilidad necesarios. Por ejemplo, si es capaz de mostrar imágenes debe prever la incorporación de una descripción textual para que pueda ser reproducida a las personas con discapacidad visual. En el caso de Xhtml esto se traduciría en la posibilidad de utilizar atributos como alt o longdesc en el código fuente de la página.
- La autoría debe ser accesible. El desarrollador debe poseer suficientes recursos/herramientas para poder diseñar contenido accesible. En el caso de Xhtml equivaldría a editores web que aporten herramientas para crear contenidos accesibles; por ejemplo, que ayuden a incluir atributos alt cada vez que el autor inserta una imagen en la página.

- El uso debe ser accesible. Los agentes de usuario, es decir las herramientas que éste utiliza, deben ser capaces de rescatar la información de accesibilidad y proporcionársela de forma conveniente. En el caso de páginas web, este apartado afecta por ejemplo a los navegadores como Mozilla, Opera o IExplorer y a los lectores de pantalla como Jaws.

Cuando en una tecnología o formato de documentos (como pdf) se cumple con estos tres requisitos se puede hablar de accesibilidad web potencial, lo que significa que al menos no existen motivos técnicos que impidan la creación de contenidos no accesibles. Esto no impli- 
ca que los contenidos creados sean realmente accesibles en todos los casos o de modo automático.

\section{Accesibilidad web y Xhtml}

Es innegable que Xhtml es el formato de base y el más extendido en la web a la hora de crear contenidos. En lo que se refiere a la accesibilidad, goza de todos los elementos necesarios para considerarlo potencialmente accesible desde varios puntos de vista:

- Tecnológico: incorpora múltiples elementos de accesibilidad.

- De la autoría: existen diferentes herramientas de desarrollo y evaluación que permiten una producción accesible. Además cada vez existen más guías y conocimiento sobre técnicas de accesibilidad.

- Del usuario: hay múltiples herramientas (navegadores y tecnologías asistivas) que ofrecen al usuario todas las características de accesibilidad del contenido.

\section{"La accesibilidad se debe asociar con todo tipo de contenido web, independientemente de cómo y mediante qué tecnologías haya sido creado"}

Seguramente debido a su amplio uso y a la multiplicidad de herramientas y técnicas de accesibilidad que giran en su entorno, no son pocos los que erróneamente asocian el concepto de accesibilidad web únicamente con Xhtml. Y esto no es así: se debe asociar con todo tipo de contenido web, independientemente de cómo y mediante qué tecnologías haya sido creado.

A continuación se va a estudiar una de las tecnologías no Xhtml más extendidas para crear y publicar contenidos en la Web, y que por estas mismas características debería cumplir con los requerimientos de accesibilidad: el formato pdf.

\section{La accesibilidad potencial del formato pdf}

Se puede decir que se ha convertido en un estándar de facto en internet. Diarios digitales, periódicos oficiales, artículos académicos, etc., no son más que unos pocos ejemplos de uso que según Adobe, la compañía que lo desarrolló, está presente en el $10 \%$ de los contenidos de la web.

Por su parte Adobe siempre ha mostrado una especial preocupación por los temas de la accesibilidad. No en vano fue una de las primeras empresas en tratar el tema incluso antes de la aparición de las primeras leyes de accesibilidad ${ }^{2}$.

A continuación vamos a revisar la accesibilidad potencial del formato pdf aplicando la lista de chequeo establecido anteriormente, esto es, analizando las posibilidades de accesibilidad de la tecnología, de sus recursos para la autoría y de sus agentes de usuario.

\section{Tecnología}

El formato pdf se inventó a inicios de la década de los 90. La inclusión de los elementos de accesibilidad aparecen por primera vez en 2001 con la versión 1.4 que incluía el producto Acrobat 5.0. Actualmente este formato puede aparecer con diferentes niveles de accesibilidad. En general se pueden considerar cuatro, partiendo de la no accesibilidad hasta el nivel máximo (potencial). Se trata de los siguientes:

- Pdf de imagen. Todo el contenido es tratado como una imagen $\mathrm{y}$, en consecuencia, es totalmente inaccesible.

- Pdf de texto. Se puede acceder al contenido textual, pero carece de informaciones necesarias para considerarse accesible, como por ejemplo el orden en el que debe ser leído. Aún así para documentos con estructuras muy simples podría tener un cierto nivel de accesibilidad.

- Pdf con marcado automático. El contenido se suministra con información adicional donde se refleja entre otras cosas toda la información de estructura y contenido del documento. Todo ello se ofrece mediante una estructura de marcas (de aquí su nombre), en formato $\mathrm{xml}$ y con importantes parecidos a html. El hecho de que el proceso sea automático implica que la accesibilidad final dependa de las características del documento y de la precisión con la que se ha añadido esta información adicional.

- Pdf con marcado manual. Normalmente consiste en un pdf que ha sido marcado automáticamente y posteriormente revisado para eliminar los posibles errores de automatización. Como era de esperar, ofrece el máximo de condiciones potenciales de accesibilidad. Por lo tanto se puede decir que el formato pdf a partir de la versión 1.4 es considerado como potencialmente accesible.

\section{La autoría}

El pdf, a diferencia de otros formatos, no tiene un editor específico por lo que el procedimiento de creación suele ser diferente al que estamos acostumbrados. En general el proceso normal ${ }^{3}$ a seguir para la creación de un documento accesible se compone de tres fases:

a. Elaboración del documento en el formato original. Esta fase inicial se puede llevar a cabo mediante un editor de textos convencional (como Microsoft Word u OpenOffice Writer). Es importante remarcar que la accesibilidad real empieza en este punto: una edición de calidad que utilice una estructura simple y bien definida y que incluya toda la información de accesibilidad necesaria, serán vitales para conseguir fácilmente un documento final accesible ${ }^{4}$. 


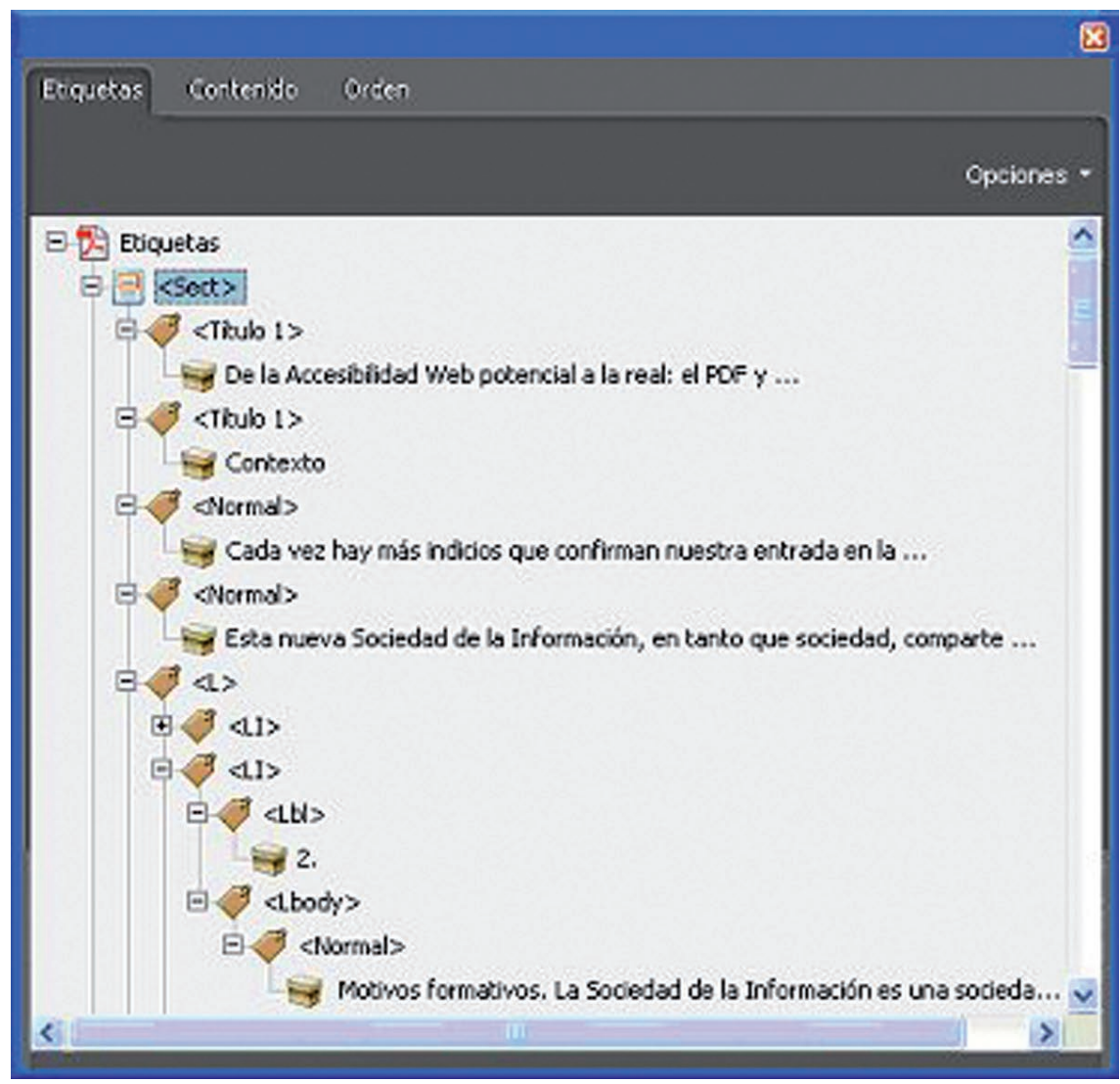

primera evaluación de la accesibilidad, este tipo de herramientas no permiten retocar el documento. Por lo tanto se hace necesario disponer de aplicaciones más sofisticadas que aparte de evaluar la accesibilidad del documento sean capaces de retocarlo. En este apartado existe un gran vacío en el ámbito del software libre, por lo que estaremos prácticamente obligados a adquirir algún producto comercial, de forma destacada la versión profesional de Acrobat.

\section{"Existen suficientes recursos y herramientas de autoría para generar documentos pdf accesibles"}

Figura 2. Estructura de un pdf marcado

b. La conversión a pdf. En este punto el programa conversor deberá ser capaz de realizar dos tareas principales:

- Recoger toda la información estructural y de accesibilidad incluida en el documento original y generar un pdf marcado.

- Inferir y asignar un orden de lectura correcto. Este proceso será especialmente importante cuando la estructura del documento sea compleja como, por ejemplo, la portada de un diario.

c. La evaluación y el retoque de la accesibilidad. Consiste en comprobar que toda la información es correcta y que el documento es plenamente accesible. En muchas ocasiones será necesario retocar o añadir información que el conversor a pdf no ha sabido o no ha podido incluir.

Como se puede observar, el proceso de autoría requiere utilizar un conjunto de herramientas específicas. En primer lugar se necesitará

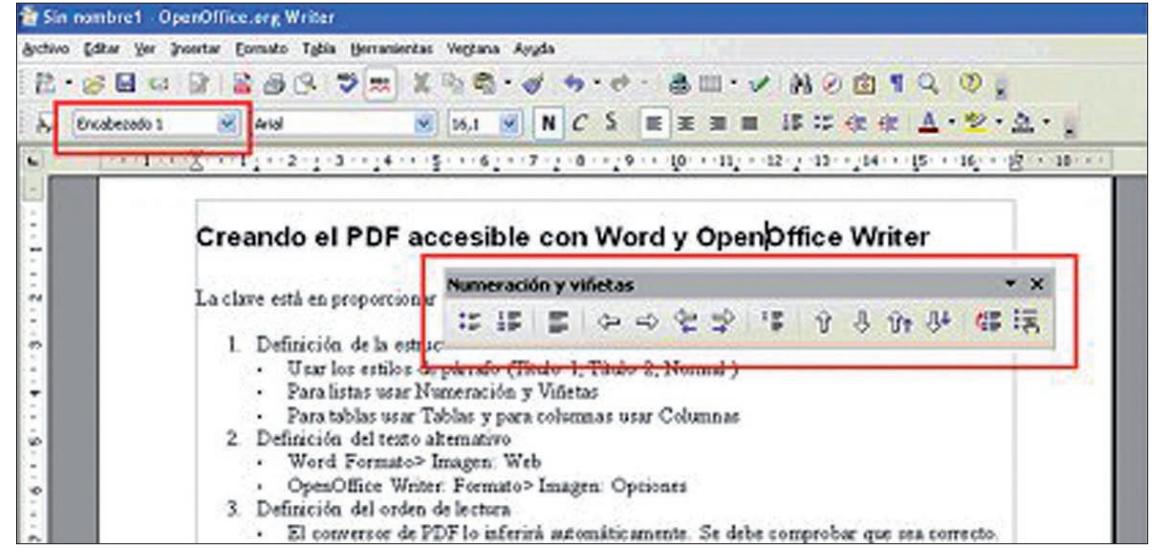

Figura 3. Un ejemplo de edición de calidad en OpenOffice

un programa conversor. Dado que los detalles de implementación del formato pdf son públicos, en este apartado existen multitud de opciones disponibles. Desgraciadamente muchos de ellos no son capaces de generar pdfs marcados y en consecuencia de crear documentos pdf accesibles.

En segundo lugar se necesitan herramientas de evaluación y retoque. A pesar de que con un simple lector de pdf se podría hacer una
A pesar de todo ello y a modo de conclusión, se puede decir que existen suficientes recursos y herramientas de autoría para generar documentos pdf accesibles.

\section{Los agentes de usuario}

Representan las herramientas que el usuario final utilizará para acceder al contenido. En el caso del pdf, es necesario evaluar dos aspectos: por un lado, la accesibilidad de los lectores convencionales de este 


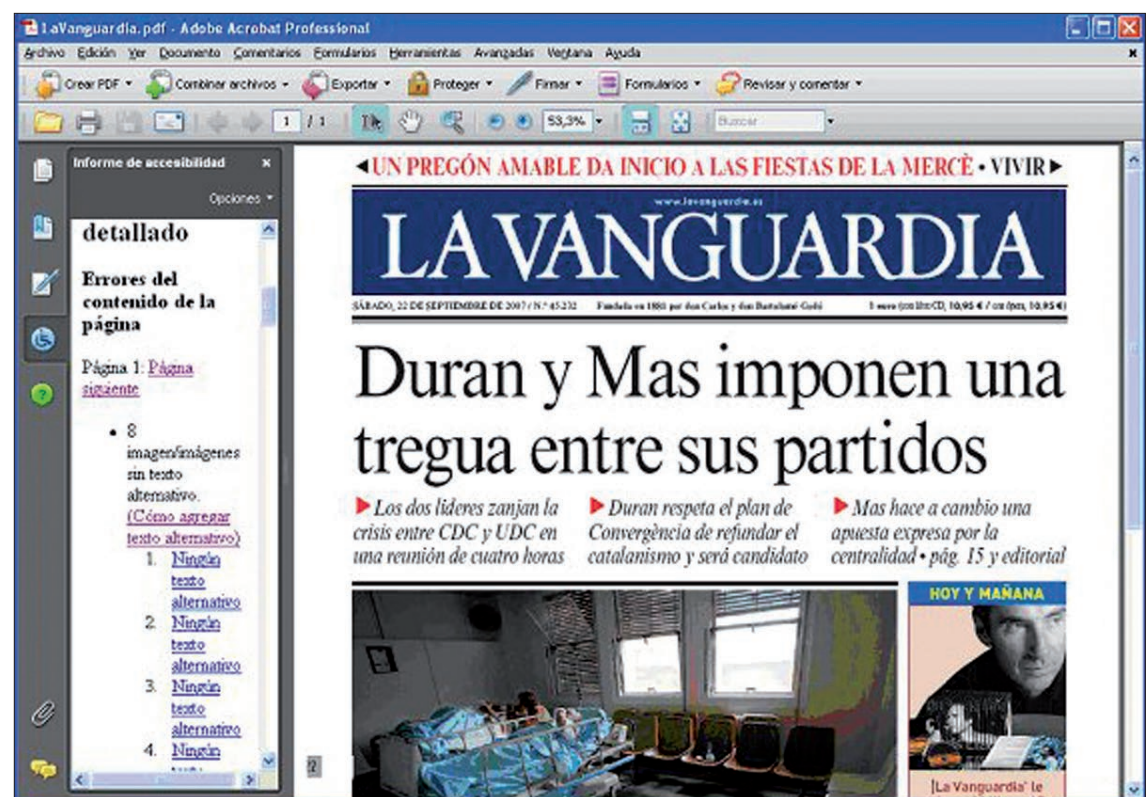

Figura 4. Evaluación de la accesibilidad en Acrobat Professional 8.0

formato, y por el otro, su compatibilidad con las tecnologías asistivas, especialmente los lectores de pantalla y magnificadores de pantalla.

En lo que se refiere a los primeros, ciertamente la herramienta más utilizada y seguramente más avanzada desde el punto de vista de la accesibilidad es el Adobe Reader. En este lector gratuito se pueden encontrar múltiples opciones de accesibilidad, como un sintetizador de voz integrado, una configuración de alto contraste o amplias posibilidades de magnificación del texto.
Respecto a la compatibilidad con las tecnologías asistivas, Adobe para Windows lleva mucho tiempo implementando la API de accesibilidad MSAA (Microsoft Active Accessibility) lo cual permite que las herramientas asistivas más importantes (como Jaws o WindowsEyes) puedan acceder a sus contenidos.

De este modo y como conclusión cabe decir que en el apartado de los agentes de usuario el formato pdf también se puede considerar accesible.

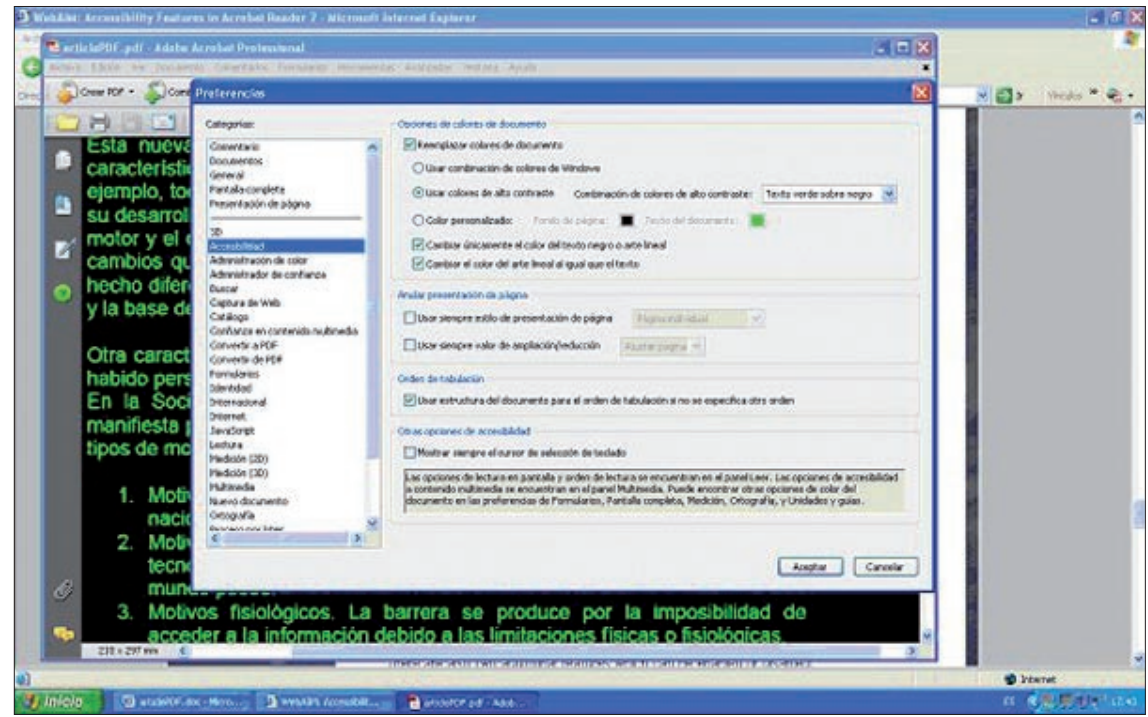

Figura 5. Accesibilidad en Reader 8.0

\section{Análisis: pdf para publicar información de actualidad en medios de comunicación españoles}

El formato pdf ha sido ampliamente utilizado en el entorno de las artes gráficas y actualmente se considera uno de los formatos más estándares y utilizados en la impresión profesional.

En el caso de los diarios digitales, se ha convertido en la pieza angular de uno de los servicios de valor añadido más usuales: la hemeroteca. Además de su objetivo puramente comercial (en la mayoría de los casos son servicios de pago y/o restringidos a suscriptores) también sirve como herramienta de archivo y preservación del patrimonio hemerográfico de la publicación.

En esta línea no es difícil ver cómo algunos diarios online han creado programas de digitalización de sus antiguos archivos en papel para convertirlos al formato pdf. Sólo como ejemplo, la hemeroteca de La vanguardia ofrece acceso a todas las ediciones impresas desde su primera publicación el 2 de febrero de 1881 .

Como se acaba de ver en los apartados anteriores, el formato pdf

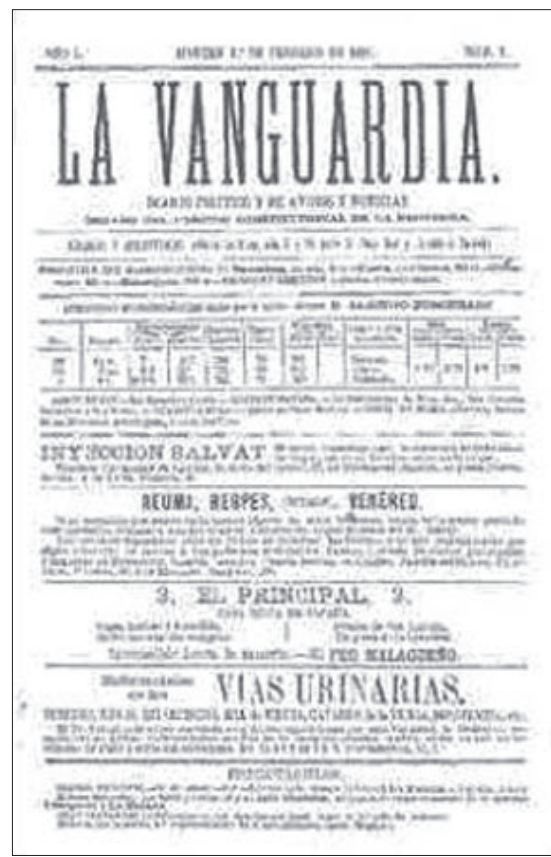

Figura 6. Primera portada de La vanguardia 
se puede considerar potencialmente accesible dado que tanto la tecnología, como los recursos de autoría y los agentes de usuario ofrecen todos los requerimientos necesarios para poder construir contenido accesible.

Sin embargo y como se comentaba anteriormente, el hecho de ser potencialmente accesible no implica necesariamente que los contenidos generados lo sean realmente.

A continuación se va a estudiar la accesibilidad potencial y real de los principales diarios digitales españoles que utilizan el formato pdf.

\section{Metodología}

Para hacer el estudio se han analizado las portadas en pdf del día 16 de noviembre de 2007 de los 25 diarios impresos de información general con una mayor difusión media entre julio de 2006 y junio de 2007, que según los datos de la Oficina de Justificación de la Difusión (OJD) son los de la tabla $1^{5}$ :

Para cada uno de los medios se han analizado los siguientes elementos:

- Nombre del conversor. Informa sobre la aplicación o librería utilizada para la creación del pdf.

- Conflicto de seguridad. Indica si en la autoría se han establecido reglas de seguridad que impiden el acceso al contenido por parte de las tecnologías asistivas.

- Versión de pdf. Para la versión del formato pdf utilizada.

- Accesibilidad potencial de la versión pdf. Indica si el formato pdf podría ser accesible. Lo será siempre que sea igual o superior a la 1.4 .

- Accesibilidad real. Indica si el pdf estudiado puede considerarse accesible. Será positivo cuando sea un pdf marcado, ya sea de forma automática o manual.
La idea y la motivación para este estudio, como se podría deducir de los apartados precedentes, es la siguiente:

- El formato pdf cumple los tres requisitos de la accesibilidad potencial. Dicho de modo aún más simple: permite crear contenidos digitales totalmente accesibles. Pero no olvidemos que esto es la accesibilidad potencial.

- Accesibilidad potencial no implica, nos guste o no, accesibilidad real, puesto que esta última depende de factores extra tecnoló- gicos, que pueden ir desde la actitud del autor hasta su déficit de formación sobre las características de la accesibilidad pasando por su implicación en la misma. Por tanto, la accesibilidad real depende también de las decisiones (o de su ausencia) de los creadores y editores de este formato.

- A partir del punto anterior, la pregunta que nos hemos formulado es la siguiente: ¿hasta qué punto las posibilidades tecnológicas son condición necesaria pero no suficiente para la eliminación de las barreras de accesibilidad?

\begin{tabular}{|c|c|c|}
\hline & Publicación & Promedio difusión \\
\hline 1 & El país & 425.927 \\
\hline 2 & El mundo & 337.172 \\
\hline 3 & $A B C$ & 230.422 \\
\hline 4 & La vanguardia & 209.735 \\
\hline 5 & El periódico de Catalunya & 177.830 \\
\hline 6 & La razón & 149.559 \\
\hline 7 & El correo & 119.114 \\
\hline 8 & La voz de Galicia & 103.702 \\
\hline 9 & El diario vasco & 84.911 \\
\hline 10 & La nueva España & 60.469 \\
\hline 11 & Diario de Navarra & 57.893 \\
\hline 12 & Heraldo de Aragón & 53.865 \\
\hline 13 & Levante-el mercantil valenciano & 44.345 \\
\hline 14 & Las provincias & 41.487 \\
\hline 15 & Faro de Vigo & 40.263 \\
\hline 16 & El diario montañés & 39.901 \\
\hline 17 & La verdad & 39.134 \\
\hline 18 & Última hora & 36.487 \\
\hline 19 & El norte de Castilla & 36.422 \\
\hline 20 & Ideal & 32.887 \\
\hline 21 & Información & 32.303 \\
\hline 22 & Diario sur & 32.293 \\
\hline 23 & La provincia & 30.990 \\
\hline 24 & Canarias7 & 30.544 \\
\hline 25 & Avui & 28.591 \\
\hline
\end{tabular}

Tabla 1. Listado de los 25 diarios con más difusión entre julio de 2006 y junio de 2007 


\begin{tabular}{|c|c|c|c|c|c|}
\hline Publicación & Conversor & $\begin{array}{l}\text { Conflicto } \\
\text { seguridad }\end{array}$ & $\begin{array}{c}\text { Versión } \\
\text { pdf }\end{array}$ & $\begin{array}{c}\text { Accesibili- } \\
\text { dad poten- } \\
\text { cial versión } \\
\text { pdf }\end{array}$ & $\begin{array}{c}\text { Accesi- } \\
\text { bilidad } \\
\text { real }\end{array}$ \\
\hline El país & PDFlib+PDI 5.0.3p1 & No & 1.4 & $\mathrm{Si}$ & No \\
\hline El mundo & Acrobat Distiller 5.0 (Win) & No & 1.2 & No & No \\
\hline$A B C$ & iText & No & 1.4 & $\mathrm{Si}$ & No \\
\hline La vanguardia & Acrobat Distiller 8.0.0 (Win) & No & 1.3 & No & No \\
\hline El periódico de Catalunya & iText & No & 1.4 & $\mathrm{Si}$ & No \\
\hline La razón & Acrobat Distiller 5.0.5 (Win) & No & 1.3 & No & No \\
\hline El correo & Acrobat Distiller 7.0.5 (Win) & No & 1.3 & No & No \\
\hline La voz de Galicia & iText 2.0.2 & Si & 1.4 & $\mathrm{Si}$ & No \\
\hline El diario vasco & Acrobat Distiller 5.0.5 (Win) & No & 1.3 & No & No \\
\hline La nueva España & Acrobat Distiller 5.0 (Win) & No & 1.4 & $\mathrm{Si}$ & No \\
\hline Diario de Navarra & Acrobat Distiller 5.0 (Win) & No & 1.3 & No & No \\
\hline Heraldo de Aragón & Acrobat Distiller 7.0.5 (Win) & No & 1.4 & Si & No \\
\hline Levante & Acrobat Distiller 7.0 (Win) & No & 1.4 & $\mathrm{Si}$ & No \\
\hline \multicolumn{6}{|l|}{ Las provincias } \\
\hline Faro de Vigo & Acrobat Distiller 5.0 (Win) & No & 1.3 & No & No \\
\hline El diario montañés & Acrobat Distiller 7.0 (Mac) & No & 1.4 & $\mathrm{Si}$ & No \\
\hline La verdad & Acrobat Distiller 7.0.5 (Win) & No & 1.3 & No & No \\
\hline \multicolumn{6}{|l|}{ Última hora } \\
\hline El norte de Castilla & Acrobat Distiller 7.0 (Win) & No & 1.4 & Si & No \\
\hline Ideal & Acrobat Distiller 7.0.5 (Win) & No & 1.4 & Si & No \\
\hline Información & Acrobat Distiller 7.0.5 (Win) & No & 1.2 & No & No \\
\hline Diario sur & Acrobat Distiller 7.0.5 (Win) & No & 1.3 & No & No \\
\hline La provincia & Ghostscript 8.54 & No & 1.4 & $\mathrm{Si}$ & No \\
\hline Canarias7 & Acrobat Distiller 7.0.5 (Win) & No & 1.6 & $\mathrm{Si}$ & No \\
\hline \multirow[t]{2}{*}{ Avui } & Acrobat Distiller 7.0.5 (Win) & No & 1.3 & No & No \\
\hline & & $\begin{array}{c}1 / 23 \\
(4,3 \%)\end{array}$ & & $\begin{array}{c}12 / 23 \\
(52,2 \%)\end{array}$ & $\begin{array}{l}0 / 23 \\
(0 \%)\end{array}$ \\
\hline
\end{tabular}

Tabla 2. Análisis de la accesibilidad potencial y real

- Por último, consideramos que para testar y poner a prueba las tres consideraciones anteriores en formatos distintos del Xhtml, los medios de comunicación españoles que publican en formato pdf podían representar un excelente banco de pruebas.

\section{Resultados}

Como se puede observar en la tabla 2, todos los diarios impresos excepto Las provincias y Última hora tienen disponible una versión en este formato. De los 23 diarios restantes, sólo uno de ellos ( $\mathrm{La} \mathrm{voz}$ de Galicia) establece restricciones de seguridad que lo hacen directamente inaccesible.

En lo que se refiere a cifras, resalta que en el $47,8 \%$ de los casos se utiliza una versión antigua y no accesible de pdf. El 52,2\% restante, aunque usan una versión potencialmente accesible, no están marcados y por lo tanto tampoco lo son.

De esta manera, ninguno de los diarios estudiados se puede considerar accesible, a pesar de que el $78,3 \%$ utiliza el conversor propio de Adobe (el Acrobat Distiller) con versiones que en principio hacen suponer que disponen de las herramientas necesarias para construir pdf accesibles. 


\section{"Ninguno de los diarios estudiados se puede considerar accesible, a pesar de que el $78,3 \%$ utiliza el conversor propio de Adobe"}

\section{Conclusiones}

En los últimos años se ha notado un importante desarrollo en la accesibilidad potencial de la gran mayoría de las tecnologías implicadas en la creación de contenidos de la web. Sin embargo, resulta frustrante ver cómo en muchas ocasiones estos avances no se reflejan en la accesibilidad real de los contenidos.

En el caso de los pdf de los diarios digitales, los resultados son alarmantes: ninguno cumple con los requisitos mínimos de accesibilidad a pesar de que en ocasiones se utilicen herramientas de autoría que sí lo permiten.

Sería interesante estudiar el porqué de este desfase. Hemos visto que en muchas ocasiones el paso de la potencialidad a la realidad depende de factores extra tecnológicos, o incluso se puede deber a cuestiones humanas como el acierto en la elección adecuada de las herramientas por parte de los autores o la formación en accesibilidad apropiada por los mismos creadores. Efectivamente, puede suceder que éstos por un déficit de formación simplemente ignoren que la tecnología posee accesibilidad potencial. Finalmente, no pueden descartarse las razones económicas: puede que los autores o editores prefieran evitar los costes adicionales (aunque sean marginales) de crear contenidos accesibles.
Por otro lado es difícil entender por qué en Adobe Acrobat la accesibilidad sea una opción y que además esté desactivada por defecto. Más aún cuando en su última versión (la 8.0) se ha mejorado el marcado automático del contenido que, a pesar de no ser el método más óptimo, permitiría sin ningún tipo de esfuerzo un nivel mínimo de accesibilidad.

La accesibilidad de un contenido disponible en la web, independientemente de su naturaleza y características, debería considerarse un requerimiento de calidad de una publicación, especialmente en los medios de comunicación, que desean aparecer ante la opinión pública como garantes de los derechos de acceso a la información de todos los ciudadanos.

\section{Notas}

1. Denominada brecha digital o, en inglés, digital divide.

2. En 1997 Adobe creó una sección de accesibilidad en su web, un año antes de que se modificara la Section 508 de EUA, una de las leyes que más impacto ha causado sobre la accesibilidad web.

3. Existen otras formas de crear documentos pdf como, por ejemplo, a partir de una imagen.

4. Existen múltiples manuales y libros que explican detalladamente los procedimientos a seguir para crear documentos pdf accesibles. Por ejemplo, el Adobe creating accessible pdf documents with Adobe Acrobat 7.0 disponible en:

http://www.adobe.com/enterprise/accessibility/ pdfs/acro7_pg_ue.pdf

5. Se ha utilizado como muestra de estudio el ranking de diarios impresos que elabora OJD y no el de diarios digitales de OJD Interactiva, porque en el primer listado aparecen todos los diarios que en sus ediciones online presentan la versión impresa en formato pdf, mientras que en el segundo, algunos diarios utilizan el formato pdf (los de ediciones impresa-digital) pero otros no (la mayoría de los exclusivamente digitales).

http://www.ojd.es/

http://ojdinteractiva.ojd.es/

\section{Bibliografía}

Adobe. Creating accessible pdf documents with Adobe Acrobat 7.0: a guide for publishing pdf documents for use by people with disabilities, 2005. Consultado en: 20-09-07. http://www.adobe.com/enterprise/accessibility/ pdfs/acro7_pg_ue.pdf

Campbell, Alastair. The four levels of pdf accessibility. Consultado en: 02-10-07.

http://alastairc.ac/2006/07/the-four-levels-ofpdf-accessibility/

Carreras, Olga. Usable y accesible: pdf accesibles. Consultado en: 02-10-07.

http://olgacarreras.blogspot.com/2007/02/pdfaccesibles.html

Clark, Joe. A list apart: articles: facts and opinions about pdf accessibility. Consultado en: 0210-07.

http://www.alistapart.com/articles/pdf accessibility

Clark, Joe. Building accessible websites. Indianapolis: New Riders, 2003.

Díaz Noci, Javier; Salaverría, Ramón (coords.). Manual de redacción ciberperiodística. Barcelona: Ariel, 2003.

Hudson, Roger. Web usability-pdf and accessibility. Consultado en: 02-09-07.

http://www.usability.com.au/resources/pdf.cfm

Lazar, J.; Dudley-Sponaugle, A.; Greenidge, K. "Improving web accessibility: a study of webmaster perceptions". En: Computers in human behavior, 2004, v. 20, n. 2, pp. 269-288.

Ribera Turró, Mireia. "Aproximación a la accesibilidad del formato pdf”. En: VIII Congreso internacional de interacción persona-ordenador, 2007, pp. 327-329.

Slatin, J.; Rush, S. Maximum accessibility: making your web site more usable for everyone, 2002 .

Thatcher, Jim, et al. Web accessibility. Web standards and regulatory compliance. Friends of ED, 2006

Thatcher, Jim, et al. Constructing accessible web sites. Peer Information Inc, 2002.

WebAIM. Accessibility features in Acrobat Reader 7. Consultado en: 02-10-07.

http://www.webaim.org/resources/reader/index. php

WebAIM. Pdf accessibility-defining Acrobat pdf accessibility. Consultado en: 02-10-07.

http://www.webaim.org/techniques/acrobat/

Agradecimientos: Trabajo parcialmente financiado por el Ministerio de Educación y Ciencia (Referencia: Proyecto HUM2004-03162/FILO).

Ramón Voces, Universidad Autónoma de Barcelona.

Ramon.Voces@uab.cat

Lluís Codina, Universidad Pompeu

Fabra.

lluis.codina@upf.edu 